\title{
La construcción de una Educación Física transdisciplinaria desde el enfoque sistémico
}

\author{
The construction of a transdisciplinary Physical Education from \\ the systemic approach
}

Nicolás Gabriel Falcettoni ${ }^{1}$ ORCID: 0000-0002-0265-8952

Recibido: 13/2/2019 • Aprobado: 29/4/2019

Cómo citar: Falcettoni, N. G. (2019). La construcción de una Educación Física transdisciplinaria desde el enfoque sistémico. Ciencia y Educación, 3(1), 11-20. Doi: https://doi.org/10.22206/cyed.2019.v3i1.pp11-20

Resumen

El presente ensayo indaga sobre la educación transdisciplinaria y cómo ella puede establecer relaciones con la Educación Física. Teniendo en cuenta las teorías complementarias como enfoque sistémico, complejidad y caos, el trabajo reflexiona, analiza y argumenta los cambios necesarios que debe implementar la Educación Física para que se adapte a las épocas actuales. Para ello, se comienza definiendo ideas y conceptos como transdisciplinariedad, sistema, pensamiento complejo y caos para, posteriormente, identificar características actuales de los elementos de la Educación Física. Por último, pretende analizar y explicar cómo, por qué y para qué se podría construir una Educación Física transdisciplinaria con base en las teorías antes mencionadas. Relacionado a ello, en el ensayo se reflexionan y se critican conceptos referidos a la Educación Física, tales como el cuerpo, el currículum, el género, la clase, los términos, la evaluación y las situaciones problemáticas del aprendizaje.

Palabras clave: Educación Física; transdisciplina; sistema; complejidad; caos.

\begin{abstract}
This essay explores transdisciplinary education and how it can establish relationships with physical education. Taking into account the complementary theories like systemic approach, complexity and chaos, the work reflects, analyzes and argues the necessary changes that the physical education must implement to adapt to the present epochs. To this end, the essay begins by defining ideas and concepts such as transdisciplinarity, system, complex thought and chaos, and then identify current characteristics of the elements of physical education. Finally, it aims to analyse and explain how, why and why a transdisciplinary physical education could be built based on the aforementioned theories. Related to this, the essay reflects and criticizes concepts related to physical education, such as body, curriculum, gender, class, terms, evaluation and problematic situations of learning.
\end{abstract}

Keywords: Physical Education; transdiscipline; system; complexity; chaos.

1. Instituto Superior de Educación Física de General Pico, La Pampa, Argentina

Correo-e: nicofalcettoni@gmail.com 


\section{Introducción}

Con el paso del tiempo muchos elementos de la vida se van modificando, ya sea para mejorar o para involucionar; la sociedad, la cultura, la política, la educación, las relaciones personales, las formas de comunicación, por mencionar algunas. Todos estos ámbitos o aspectos que van sufriendo variaciones se afectan entre sí, de manera recíproca. Por ejemplo, si las sociedades se modifican, son inminentes y paralelas las mutaciones en la educación, y dentro de ella, las distintas áreas curriculares que conforman los núcleos de aprendizaje. La Educación Física no está exenta a las reformas y a todos aquellos componentes que rodean a las personas, por lo tanto, en un mundo donde todo cambia en poco tiempo, y cada vez se complejiza más, se necesitan enfoques educativos nuevos que logren sintonizar con la realidad actual. La raíz de esta concepción la confirma Pérez (2003):

El Paradigma de la Complejidad aglutina a científicos de diversos campos de conocimiento que insisten en la conveniencia de adoptar nuevos modelos teóricos, metodológicos y, por ende, una nueva epistemología, que permita a la comunidad científica elaborar teorías más ajustadas de la realidad que posibilite, al mismo tiempo, diseñar y poner en prácticas modelos de intervención - social, sanitaria, educativa, política, económica, ambiental, cultural, etc.más eficaces que ayuden a pilotar y regular las acciones individuales y colectivas. (p. 2)

En concordancia con lo anterior, es indispensable pensar y construir, desde un enfoque sistémico, una Educación Física que sea transdisciplinaria. Por lo tanto, el presente ensayo buscará refutar las reproducciones y tensiones de una Educación Física tradicional, unidisciplinaria y acrítica, con la finalidad de argumentar los nuevos cambios necesarios y comenzar a construir una transdisciplinaria. Ahora bien, en primer lugar, debemos definir conceptualmente la Educación Física, ya que, si bien es una asignatura escolar, dependiendo desde qué enfoque se analice y qué finalidades persiga, su significado será diferente. Tradicionalmente, desde una perspectiva cartesiana, esta asignatura dedicó su tiempo a educar los cuerpos fisiológicos de los alumnos, es decir, fomentó la formación y desarrollo motriz de un cuerpo máquina. En este sentido, Soto y Vargas (2019) afirman:

La visión mecanicista y técnica que aún prevalece en el ámbito de la Educación Física y las estrategias que los sustentan, eluden al ser humano como ser íntegro y como una unidad, al considerar el cuerpo como objeto o sumatoria de partes. (p. 416)

Por ello, si se toma como referencia el término Educación Física, el análisis se reduce a la educación de cuerpos anatómicos y mecánicos; estableciéndose un campo educativo limitado para los tiempos sociales vigentes. Por otra parte, existen autores que reflexionan sobre su aplicación actual en las escuelas. Lavega (2018) expresa que la asignatura "no pretende enseñar únicamente a botar un balón, (...) sino también a educar a toda la persona que interviene mientras está participando (...) busca ejercer una influencia sobre las personas a través de su participación en distintas prácticas motrices” (p. 74). En efecto, la Educación Física va más allá de la mera enseńanza mecánica, por lo tanto, se encuentra atravesada por la cultura y el movimiento, donde la motricidad cobra cierto sentido para cada sujeto. Es así que Gallo (2016) sostiene:

(...) la educación física empieza a preguntarse por conceptos que hasta entonces habían sido poco estudiados como la corporeidad, la motricidad y la intencionalidad. Por ejemplo, el concepto de movimiento empieza a ser problematizado porque ya no se refiere a la visión mecanicista del cuerpo, sino a la motricidad como acciones y expresiones intencionadas. (pp. 134-135)

Entonces, la Educación Física es un área que puede ser definida desde distintos enfoques, sin embargo, debe adecuarse a las complejidades del mundo actual y lograr construirse desde la transdisciplinariedad.

A pesar de que algunos investigadores están indagando hoy sobre la transdisciplina, el concepto tiene antecedentes en Piaget, según lo afirma Martínez Miguélez (2007):

Aunque la idea central de este movimiento no es nueva (Piaget la proponía ya en los años 70 como 
una "etapa nueva” del conocimiento), su intención es superar la parcelación y fragmentación del conocimiento que reflejan las disciplinarias particulares y su consiguiente hiperespecialización, y, debido a esto, su incapacidad para comprender las complejas realidades del mundo actual, las cuales se distinguen, precisamente, por la multiplicidad de los nexos, de las relaciones y de las interconexiones que las constituyen. (p. 2)

Por último, el artículo desarrollará conceptos como los sistemas, la complejidad y su paradigma, caos, la crisis del término Educación Física, qué encierra el concepto de la transdisciplina y cómo podría pensarse y construirse una Educación Física desde el enfoque sistémico.

\section{Transdisciplina, sistema, caos y complejidad}

(...) por educación transdisciplinaria se asume aquí esa multifacética dimensión de la educación, que fundada en la integración de los paradigmas emergentes, se orienta a la promoción, divulgación y facilitación metacognitiva de conocimientos, habilidades y capacidades, así como al cultivo del espíritu humano en el diálogo entre educador y educando (junto a otros actores), quienes discuten, se contraponen y complementan, tanto con razón como con pasión, sobre la búsqueda de soluciones a problemas complejos. Es un nuevo tipo de educación que se fundamenta en el enfoque integrador de la perspectiva dialéctico-compleja, que debe ser fomentado por educadores, instituciones, actores sociales y medios de difusión masiva con el fin de facilitar y promover la apropiación de esos valores por todos los ciudadanos. (Ardoino, citado por Luengo y Álvarez, 2018, p. 79)

Para Nicolescu, citado por Delgado y Rist (2016): "la transdisciplinariedad es relevante para todo lo que se halla entre disciplinas, así como lo que atraviesa y traspasa las disciplinas" (p. 40). A partir de aquí, hablar de transdisciplina es entonces trascender a las disciplinas. Por este motivo, para trascender de las mismas, se debe hablar de teorías que la complementan; profundizando el concepto y promoviendo la integración holística de varias teorías que permitan sustentar la idea transdisciplinaria. Una de esas teorías, y la que primero deberíamos nombrar, es la "Teoría general de los sistemas". Afirma, entre otros conceptos, que el todo es más que la suma de las partes. Ya en la antigüedad, Aristóteles fomentó una idea que serviría de base para siglos posteriores. Según Luengo y Martínez (2018):

(...) el hecho de percatarse de la importancia capital del entorno o contexto, donde se enriquecen de manera infinita las cosas, fue lo que condujo a Aristóteles a formular su famosa idea simiente del enfoque sistémico: el todo es más que la suma de sus partes, es decir, supone reconocer no solo los nexos y las interacciones, sino "lo nuevo" que resulta de ello. (p. 28)

Tomando esta premisa, el biólogo austriaco Ludwig Von Bertalanffy enriquece la concepción, y comienza a sostener que los organismos vivos se encuentran en un sistema donde constantemente intercambian sustancia y energía con el ambiente (Luengo y Martínez, 2018, p. 28). Esta idea, también empieza a ser aplicada por pensadores de otras áreas como la matemática, física, psiquiatría, educativa, entre otras. En resumen, los sistemas se definen como "conjuntos de elementos que guardan estrechas relaciones entre sí, que mantienen al sistema directo o indirectamente unido de modo más o menos estable y cuyo comportamiento global persigue, normalmente, algún tipo de objetivo" (Arnold y Osorio, 1998, p. 41).

Partiendo de esta idea, no basta con analizar un objeto, un hecho o un ser vivo desde el enfoque sistémico; sino que debemos penetrar el sistema y comprender qué es lo que puede suceder dentro. Cada elemento que integra el sistema se encuentra en conexión con otros que cooperan para que el sistema funcione en su totalidad. Entonces, si cada uno de ellos funciona siempre de la misma manera estamos hablando de un sistema rígido y cerrado; pero si coexisten hechos que perturban y desordenan el "normal" funcionamiento del sistema, hablaríamos de un sistema dinámico y abierto. Estos fenómenos pueden ser comprendidos desde la "Teoría del caos". 
La "Teoría del caos" busca explicar que en determinados momentos surgen "de la nada" hechos inexplicables que alteran el orden, en este caso de un sistema. Retomada de las ideas iniciales de Poincaré, Edward Lorenz, (meteorólogo y matemático estadounidense) al observar que le resultaba impredecible pronosticar el clima con gran extensión de días, afirmó que "pequeños cambios en las condiciones iniciales producían diferencias asombrosas (...) en el resultado, con lo cual las predicciones meteorológicas a mediano o largo plazo resultaban imposibles" (Cazau, 1995, p. 5).

Otra teoría que nos puede ayudar en la ulterior construcción transdisciplinaria de la Educación Física es la "Teoría de la complejidad o pensamiento complejo”. La misma, sostiene que:

Todo conocimiento opera mediante la selección de datos significativos y rechazo de datos no significativos: separa (distingue o desarticula) y une (asocia, identifica); jerarquiza (lo principal, lo secundario) y centraliza (en función de un núcleo de nociones maestras). Estas operaciones, que utilizan la lógica, son de hecho comandadas por principios "supra lógicos" de organización del pensamiento o paradigmas, principios ocultos que gobiernan nuestra visión de las cosas y del mundo sin que tengamos conciencia de ello. (Morin, citado por Burgos, Salvador y Narváez, 2016, p. 146).

Pensar y enfocar a la Educación Física en el mundo actual, debe contribuir a mejorar la educación y, al mismo tiempo, la práctica docente. En efecto, las complejidades de las relaciones sociales obligan a abordar la asignatura escolar desde la transdisciplina, ya que hoy es más que aquella disciplina cartesiana.

\section{Transdisciplina y Educación Física ¿ecómo re- lacionar las distintas teorías con la Educación Física?}

Para comenzar, debemos reflexionar y analizar cómo la transdisciplinariedad y las diversas teorías nombradas influyen en la Educación Física.

Las personas estamos constantemente interconectadas con todo lo que nos rodea. No podemos reducirnos y analizarnos como seres aislados desde un enfoque positivista, ya que somos sociales y nos encontramos inmersos en una realidad que se define como un todo organizado o sistema.

Cuando se observan las conexiones entre el microcosmos y el macrocosmos se percibe que el ser humano no está en medio del caos y de la arbitrariedad, sino que pertenece a una extensa red de interdependencias, complementaciones y reciprocidades que constituyen la vida (Collado Ruano, citado por Ruano, Morillo y González, 2018, pp. 619-620).

Esta visión holística de los fenómenos permite analizar aquellos cambios que se producen dentro de un sistema organizado y que, a su vez, generan modificaciones impredecibles, es decir, hay un caos que altera el orden. Tal como afirma Duque (2017):

La teoría del caos plantea que el mundo no sigue un patrón fijo y previsible, sino que se comporta de manera caótica, y que sus procesos y comportamiento dependen, en gran manera, de circunstancias inciertas. (...) una pequeña variación en el sistema o en un punto de este puede provocar que en un lapso de tiempo a futuro este presente un comportamiento completamente diferente e impredecible. Este concepto va ligado a la teoría de sistemas. (p. 141)

Lo mismo sucede con las ciencias de la educación en todas sus dimensiones, es decir, no deben comprenderse desde su parcela epistemológica, sino innovar y adaptarse a los avatares de la realidad. Si enseñar Educación Física no va por el mismo camino, los profesionales del área estaremos siendo incoherentes con la dinámica integradora, propia y de nuestros alumnos.

Pero ¿qué aspectos de la Educación Física se pueden sustentar en las teorías mencionadas? Considero que la relación es amplia, no obstante puedo brindar algunos ejemplos. Si tomamos en cuenta a la Educación Física per se, podemos decir que es un sistema. Es decir, el paso del tiempo con los cambios de paradigmas, teorías de aprendizaje, pedagogía, didáctica, currículo, concepción de cuerpo y de sujeto, la cultura y la motricidad son elementos que han ido incorporándose en la vida de la Educación Física y cada uno, en conexión con los demás, hace 
funcionar a este sistema denominado Educación Física. Sin embargo, el término peca de obsoleto, ya que no es holístico ni integrador, sino más bien reduccionista, rutinario y sintetizador.

Esto supone un cambio urgente en el nombramiento del área (por lo pronto en aquellos países que aún conservan el nombre tradicional). Hoy sabemos que la Educación Física trabaja con las prácticas corporales y motrices que pertenecen a una cultura determinada, por este motivo concluyo que el término Educación Física ha quedado anticuado. Si se modificara por un término más global, amplio y holístico estaríamos en presencia de un paso gigante en la concepción de la Educación Física transdisciplinar.

La palabra educación refiere, según García Carrasco y García del Dujo, citados por Navas (2004), a:

"criar" y "crianza", que hacían alusión a "sacar hacia adelante", "adoctrinar" como sinónimo de "doctrino", y "discipular" para indicar "disciplina" o "discípulo". Son términos que se relacionan con los cuidados, la protección y la ayuda material que dedicaban las personas adultas a los individuos en proceso de desarrollo. (p. 31)

En cuanto a "física", es obvio que el concepto no alude a aquella ciencia que estudia la materia y energía, a través de leyes, sino que se reduce estrictamente a lo corporal. Por lo tanto, se habla de una formación o "crianza" del cuerpo o una educación del cuerpo; un concepto que se asocia con el dualismo cuerpo-mente. Ahora bien, aquí tenemos otro inconveniente ¿qué concepción del cuerpo tenemos? ¿Es un cuerpo máquina visto desde las ciencias exactas, o es una unidad orgánica, donde todos los sistemas se relacionan y un cuerpo es algo que siente, piensa y se mueve? ¿Tiene incidencia la cultura, la sociedad y las relaciones en ese cuerpo? Todas estas cuestiones hacen a la Educación Física caótica. En algún momento estaba ordenada, pero las diversas investigaciones y críticas hicieron que se replanteen varias aristas.

Por otra parte, si planteamos mejoras en los contenidos y los adaptamos según las realidades culturales de los alumnos podemos concluir que la Educación Física también es compleja, ya que lo que se observa y se expresa en una clase tiene directa relación y conexión con la vida social de cada individuo. Los sujetos no están separados de su sociedad cuando ingresan a la escuela, por ende, los objetivos del área deben estar directamente relacionados con el mundo que nos rodea. Para Pastor, Brunicardi, Arribas, y Aguado (2016) la Educación Física debe ocuparse de los siguientes aspectos: "el desarrollo físico-motriz del alumnado; la creación y recreación de la cultura física del alumnado; y su aportación al planteamiento global de desarrollo integral del alumnado, como ciudadanos de una sociedad democrática” (p. 183). Nótese que los autores ya mencionan el término "cultura física”, introduciendo una idea de enseñanza más holística, basada en el sentido amplio de cultura, y no "educación física" nombre que, como se ha expuesto, resulta conceptualmente restrictivo.

Para resumir, se podría decir que, desde un enfoque sistémico, la Educación Física contiene elementos que interactúan entre sí (paradigmas, teorías del aprendizaje, cultura, pedagogía, currículum, género, situaciones problema, evaluación, y muchos más); es caótica, ya que se desordena cuando se producen hechos o fenómenos impredecibles (aquellos que suceden en una clase), y es compleja porque no es independiente, sino que está inmersa en el mundo de la cultura y las relaciones sociales.

\section{La construcción de una Educación Física transdisciplinaria}

La educación, y más precisamente la Educación Física, se limitó a enseñar sus saberes a lo largo de la historia. Cambiando de paradigmas, contenidos o concepciones del cuerpo, es un área en constante tensión y fluctuación. Cada actor que interviene intenta imponer una ideología de la Educación Física.

Si analizáramos este campo desde la transdisciplinariedad observaríamos que cada actor es un componente que aporta su idea o su parecer; sin embargo, distinto sería mirar al interior del campo y quedarse solo con uno de esos actores. Esto ha sido el historial de la Educación Física, es decir, las políticas educativas o quienes tienen la autoridad e idoneidad para conformar un currículo, han instalado modas o discursos según las investigaciones emanadas. Delgado y Rist (2016) permiten sustentar esta idea: 
Un desafío importante para la transdisciplinariedad consiste en hallar formas de estimular un diálogo y una cooperación entre grupos heterogéneos de actores sociales con distintas formas de conocimiento, en vez de imponer una sola visión 'coherente' del mundo, a través de un discurso hegemónico que silencie a todos los demás discursos y se coloque fuera del objeto del análisis. (p. 41)

Abordar el campo de la Educación Física desde la transdisciplina podría ser uno de los pasos que nos permitiera acercarnos y comprender por qué este espacio es como es.

Ahora bien ¡cómo deberíamos construir una Educación Física transdisciplinaria? Una de las ideas que postulé anteriormente fue la modificación del término o del nombre del espacio por otro que sea más integrador y que nos permita asociar varios elementos, entre ellos la cultura. Pero con eso no bastaría, ya que durante las interacciones docente-alumnos entran en juego otros elementos que pueden ser analizados desde lo sistémico.

En una clase, aquellas prácticas corporales y motrices que se enseñen pertenecen a la cultura en la cual están inmersos los alumnos, los docentes y la escuela. Por este motivo, desde un principio, sabremos que la heterogeneidad y realidades de nuestros alumnos harán que cada una de las clases sea compleja. Cuando el docente plantea un contenido puede haber resistencia, dificultad de resolución o simplicidad en otros, conocimiento o no del contenido; al mismo tiempo, pueden surgir respuestas motrices que tal vez no se habían imaginado al elaborar la planificación; sin embargo, la clase debe funcionar como un sistema dinámico, ya que van a ocurrir diversos hechos que siempre estarán relacionados con la vida real de cada sujeto, no solo del conjunto de alumnos, sino también del docente que participe. Por ende, los aspectos sociales y culturales entran en juego constantemente, al igual que el pensamiento complejo, ya que este "importa una forma de pensar y, a la vez, de estar en el mundo, razón por la cual la cognición y el valor están indisolublemente ligados" (Francisco y González, 2017, p. 419). Paralelamente, las tareas planteadas deben estar en sintonía, no solo con la cultura, sino también con las necesidades del mundo actual, que al mismo tiempo es un sistema caótico y complejo.

Otra de las cosas que sucede en las clases de Educación Física es la estructuración de un formato clase que pareciera debe ser respetado sí o sí. Desde ya, en las épocas que vivimos, es imposible querer mantener una disciplina en los alumnos, en cuanto a que ya no somos aquel profesor que era modelo y solamente dirigía, sino que debemos dejar que los alumnos se expresen con todos sus sentidos. Querer mantener un orden, es ignorar la riqueza que nos puede dar el desorden. Por esto, el caos aparece en muchos momentos y, desafortunadamente, los docentes lo reprimen. Arboleda (2016) sostiene que:

La Teoría del Caos se centra en la construcción del conocimiento, permitiendo que dicha construcción se dé en los puntos de encuentro entre teoría y práctica, colocando a la práctica como la fuente principal de conocimiento. El aprendizaje como sistema o subsistema caótico de la Escuela se da a través del encuentro de las relaciones entre actividades, situaciones problema, etc., que se organizan bajo la dialógica permanente entre orden y desorden, con procesos permanentes de construcción de conocimiento sobre un camino de incertidumbres. (p. 98)

Tal vez el alumno que está realizando la tarea de otra manera puede decirnos mucho u orientarnos a mejorar la práctica, por ello, cada tarea plateada debe significar una situación problema a resolver. Es allí donde se da la multiplicidad de aprendizajes, o como afirma Collom Cañellas, citado por Arboleda (2016): "cabe entender la creatividad como el caos de la inteligencia, es decir, como la consecuencia de la no linealidad del cerebro" (p. 98). Este caos que vengo analizando también forma parte de la diversidad de sujetos que se encuentran en una clase. Para que la Educación Física sea transdisciplinaria debe comprometerse, no solo desde su explicitación en los documentos o currículos, sino también desde la aceptación de la diversidad y la heterogeneidad en las clases. Un logro que se ha alcanzado en muchos lugares ha sido la implementación de las clases mixtas, que aún se encuentra bajo las reproducciones 
conservadoras de muchos docentes que afirman que no se pueden "mezclar" varones con mujeres porque los primeros tienen más fuerza, reduciendo el discurso a mecanicista, positivista y discriminador. Sobre esto me pregunto ¿asisten a la Educación Física para medir fuerza o para aprender? Y repregunto ¿en la sociedad no viven mujeres y hombres, o cada género tiene su espacio geográfico? ¿La escuela, y precisamente la Educación Física, no debieran formar la condición humana?

El currículo, entonces, es otro elemento que nos puede acercar a la Educación Física transdisciplinaria. Rodríguez (2017), en referencia al currículo, sostiene que "es un sistema abierto, ya que se entiende en la incertidumbre y se reconoce en la diversidad, contribuyendo en su regeneración y, a su vez, es regenerado por ella” (p. 428). Ligado inevitablemente a la cultura, el currículo permite un primer acercamiento a los contenidos y recomendaciones explícitos, sin embargo, se sabe que muchas veces los docentes prefieren adoptar enfoques tradicionales y no pensar a las clases como complejas y diversas. Esto se observa cuando, a pesar de la modalidad mixta, los docentes separan a varones y mujeres para proporcionarles contenidos diferentes; o también cuando solo se realizan deportes durante todo el ciclo lectivo. De esta manera continúan reproduciéndose modelos hegemónicos, tradicionales, deportivos y competitivos de la Educación Física.

Díaz de Greñu Domingo y Anguita Martínez (2017) sostienen que: "Los estereotipos en torno al género y a la orientación sexual que han predominado conducen, pues, a la discriminación y el sistema educativo desempeña una función clave en la transmisión y reproducción de los mismos" (p. 222). Ante esto, cabe reflexionar: los docentes que llevan adelante clases con separación de género y/o competitividad, que reproducen este modelo o manejan este discurso separatista y diferenciador de manera imperante ¿considerarán que la Educación Física solo sirve para los aptos motrizmente o para varones? ¿Y a los demás, no les reportará nada? Más adelante las mismas autoras agregan:

(...) aunque el profesorado negaba poseer sesgos de género, se apreciaban diferencias de trato, se hacían bromas sexistas, se producían comentarios basados en estereotipos, se adjudicaban determinadas tareas dependiendo del sexo, había negligencia hacia ciertos comportamientos agresivos y se fomentaba la competitividad en los chicos y la pasividad en las chicas. (Díaz de Greñu Domingo y Anguita Martínez, 2017, p. 224)

Nuestra Educación Física debe ir más allá (transdisciplinaria), enseñando valores y prácticas culturales a sujetos que son iguales y distintos al mismo tiempo. Iguales, porque la educación debe fundamentarse en la ética y en valores como la equidad y el respeto, no en simpatías o afinidades del docente. Distintos, porque cada individuo es único; tiene sus propias características, habilidades, experiencias y necesidades. Todos tienen derecho a aprender los mismos contenidos.

La evaluación es otro aspecto que puede analizarse desde la transdisciplinariedad. La Educación Física se ha caracterizado por tomar las palabras “medición y evaluación” como sinónimos por mucho tiempo, incluso en la actualidad, por lo tanto, las calificaciones finales dependían siempre de distancias, tiempos, técnicas y habilidades. Por ello, según Martín et al. (2006): "puede observarse la aplicación del mundo del deporte a la EF, puesto que a la hora de la evaluación (calificación) el profesor (entrenador) desempeña funciones de juez deportivo. Estas funciones son: medir, cronometrar y tabular resultados" (p. 33). En conclusión, no se valora a los alumnos como sujetos con pensamientos complejos pertenecientes a una sociedad, sino como actores que deben cumplir requisitos físicos. Sin embargo, gracias a muchas investigaciones, sabemos que no es el docente quien solo puede evaluar, sino que los alumnos también tienen derecho a hacerlo. No existe una solo forma de evaluar.

El término evaluar refiere a "formular un juicio de valor sobre el desempeño” (Ravela, 2016, p. 44); en este sentido, Ortiz Hernández (2007) expone: "La evaluación es uno de los constituyentes del proceso de enseńanza-aprendizaje $y$, por ende, contribuye a que el estudiante aprenda" (p. 108). Entonces, si pensamos que el aprendizaje se construye y no se impone, este "juicio de valor" corresponde a la creación de datos a analizar a partir de la información 
recogida anteriormente, ya sea en una o varias clases. No es pura y exclusivamente del docente, sino que hay otras maneras que permiten poner a los alumnos en un lugar de sujetos activos, reflexivos, autocríticos, críticos y democráticos. Estos conceptos no escapan a la vida social de cada uno de los educandos, sino que se asemeja a los factores y dificultades que se enfrentan todos los días. Por esto, la evaluación debe funcionar como un sistema y ser, a su vez, un componente del mismo. Es decir, un juicio de valor (evaluar) no se origina mecánicamente al observar una distancia o un tiempo, sino que se construye a partir de varios elementos que se van considerando.

Algunos ejemplos de tipos de evaluación que debiera contemplar la Educación Física transdisciplinaria: a) autoevaluación: tanto los docentes como los alumnos pueden reflexionar y emitir una autocrítica; b) evaluación formativa: se optimiza el proceso de enseñanza-aprendizaje desde una perspectiva humanizadora (Red de Evaluación Formativa y Compartida, citada por, Hamodi, López Pastor, V y López Pastor, A, 2015, p. 150) y c) coevaluación o evaluación compartida: la evaluación se genera entre los pares.

Retomando los conceptos de complejidad y caos, el primero y el último tipo de evaluación se conectan con los pensamientos humanos y los hechos impredecibles, ya que para Bocanegra (2014) este par de juicios de valor "permite revisar con una óptica humanística y social, sus contenidos centrados en el ser humano y convierte la coevaluación y auto evaluación en herramientas en procesos de mayor riqueza en su pertinencia y contextualización" (p. 35).

Por último, creo necesario que los docentes sepamos explicarles a nuestros alumnos por qué y para qué les puede ser útil lo que están aprendiendo. $\mathrm{Al}$ mismo tiempo, el grupo de alumnos debe tener la posibilidad de poder expresar su disconformidad o moldear los contenidos. El planteamiento de situaciones problemáticas se entrelaza con la complejidad y el caos, al mismo tiempo que, para resolver una tarea se pueden encontrar miles de alternativas. Esto es así porque nuestros sentidos, experiencias, vivencias y aprendizajes (nuestro cuerpo como sistema) nos brindan la capacidad de reflexionar y buscar la mejor respuesta, sin embargo, también podemos aprender de los demás, y es allí donde lo caótico es bueno y lo complejo nos puede ser simple.

A veces estas situaciones precisan ser resueltas de forma individual o conjunta. En este último caso, las tareas que proponen cooperación son las más acertadas (un valor que histórica y políticamente se deja de lado), aunque lo importante de la propuesta sigue siendo la incertidumbre de la resolución. Para este concepto cito a Mendoza Molina y Bernabeu Tamayo (2006), que se refieren al tema como aprendizaje basado en problemas:

El papel que los estudiantes asumen en el método ABP, es el de ser protagonistas en la resolución del problema, ellos se hacen cargo de la situación. (...) este conjunto de actividades promueven $[$ sic] alumnos reflexivos, críticos, creativos, $\mathrm{y}$ los adentra en el campo de la metacognición, es decir, aprenden como aprender mejor y, por lo tanto, pueden elegir de que [sic] manera hacerlo. Además se estimula la investigación porque, en realidad, es lo que están haciendo. (Sección ABP y la formación de estudiantes, párr. 8-9)

En una Educación Física transdisciplinaria, las situaciones problemas colocan a los alumnos en circunstancias reales que se pueden asemejar a lo que atraviesan durante su vida, es decir, resolver problemas constantemente.

\section{Conclusiones y recomendaciones}

Una Educación Física transdisciplinaria debe estar sustentada en el enfoque sistémico. Así, nos permitirá avanzar sobre las complejidades del mundo y, más precisamente, en los alumnos. Pero para que el área pueda ser analizada como transdisciplina, debe modificar ciertos elementos:

- Pensarse como un sistema dinámico abierto que contemple elementos como la cultura, la sociedad, la clase, la pedagogía, las políticas educativas, etc. A partir de allí, diseñar clases (que también son sistemas) que sean significativas para la cultura del estudiante.

- Cambiar el término Educación Física por otro que integre la motricidad cultural. 
- Eliminar toda clase de discriminación de género, con el objetivo de incluir y tratar a todos por igual; ya que en la sociedad vivimos e interactuamos todos juntos (somos un sistema).

- Modificar la evaluación directa y calificativa por otras que realmente cumplan la finalidad de emitir un juicio de valor, ya sea propio o ajeno.

- Crear tareas con base en situaciones problemáticas que produzcan caos e incertidumbre para que los alumnos piensen, complejicen, busquen, experimenten y resuelvan solos o con otros los escenarios planteados. Esa es la forma de aprender desde la formación y no desde la instrucción.

- Permitir que exista el caos y desorden para que se construyan nuevas alternativas de aprendizaje. La Educación Física no debe reprimir las expresiones y creaciones de los alumnos.

- Reconocer y abordar el análisis de otros elementos que pueden interaccionar en la Educación Física.

- Profundizar la investigación y el perfeccionamiento docente para que la Educación Física esté en sintonía con las realidades sociales.

- Incorporar otras teorías que amplíen el campo transdisciplinar de la Educación Física.

- Para educar desde la transdisciplina, se debe promover la formación docente transdisciplinar.

Preguntas para reflexionar:

¿Qué otros elementos de la Educación Física se podrían modificar para que finalmente sea transdisciplinaria? ¿Qué modificaciones necesitaría el sistema educativo para incluir una Educación Física transdisciplinaria?

\section{Referencias}

Arboleda, A. S. (2016). La escuela bajo los preceptos de la teoría del caos: incertidumbre, caos, complejidad, lógica difusa y bioaprendizajes. Biociencias, 11(1), 91-103. Recuperado de: https://dialnet. unirioja.es/servlet/articulo? codigo $=5646114$

Arnold, M y Osorio, F. (1998). Introducción a los conceptos básicos de la teoría general de sistemas. Cinta de moebio, (3), 40-49. Recuperado de: http://www.moebio.uchile.cl/03/index.html
Bocanegra, S. S. (2014). Auto y coevaluación, complementariedad significativa en la evaluación de las Ciencias Sociales. Escenarios, 12(1), 34-49. Recuperado de: https://dialnet.unirioja.es/s ervlet/ articulo? codigo $=4763466$.

Burgos, J. B., Salvador, M. R. A., \& Narváez, H. O. P. (2016). Del pensamiento complejo al pensamiento computacional: retos para la educación contemporánea. Sophia: Colección de Filosofía de la Educación, (21), 143-159. doi: 10.17163/ soph.n21.2016.06.

Cazau, P. (1995). La teoría del caos. Recuperado de http://galeon.com/pcazau/artfis_caos. htm.

Delgado, F., \& Rist, S. (2016). Las ciencias desde la perspectiva del diálogo de saberes, la transdisciplinariedad y el diálogo intercientífico. Recuperado de https://boris.unibe.ch/91492/1/Rist_2016_ las $\% 20$ ciencias $\% 20$ desde $\% 201 a \% 20$ perspectiva\%20del\%20dialogo.pdf.

Díaz de Greñu Domingo, S. \& Anguita Martínez, R. (2017). Estereotipos del profesorado en torno al género y a la orientación sexual. Revista Electrónica Interuniversitaria de Formación del Profesorado, 20(1), 219-232. doi: https://doi.org/10.6018/ reifop/20.1.228961.

Duque, Ó. S. (2017). Mirada de la Gestión moderna desde la teoría del caos y la transdisciplina. Universidad \& Empresa, 19(33), 137-161. Recuperado de: https://revistas.urosario.edu.co/ xml/1872/187251252006/187251252006_visor_jats.pdf.

Francisco, T. G., \& González, J. R. (2017). Complejidadeducación: un aporte para las generaciones futuras. Educación y Humanismo, 19(33), 409-424. doi: http://dx.doi.org/10.17081/eduhum.19.33.2653.

Gallo, L. E. (2016). Variaciones sobre la motricidad en la educación del cuerpo. Impetus, 10(2), 63-74. Recuperado de: http://revistaimpetus.unillanos. edu.co/impetus/index.php/Imp1/article/view/171.

Hamodi, C., López Pastor, V. M., \& López Pastor, A. T. (2015). Medios, técnicas e instrumentos de evaluación formativa y compartida del aprendizaje en educación superior. Perfiles educativos, 37(147), 146-161. Recuperado de: http://www.scielo.org. mx/pdf/peredu/v37n147/v37n147a9.pdf. 
Lavega, P. (2018). Educar conductas motrices: reto necesario para una educación física moderna. Acción motriz, 2018, (20), 73-88. Recuperado de: https://repositori.udl.cat/handle/10459.1/64795.

Luengo, N. A. \& Martínez Álvarez, F. (2018). La educación transdisciplinaria. Buenos Aires, Argentina: Comunidad Editora Latinoamericana.

Martín, J. J. B., Aguado, R. M., García, J. G., Pastor, E. M. L., Pinela, J. F. M., Badiola, J. G., ... \& Martín, M. I. (2006). La Evaluación en Educación Física: revisión de modelos tradicionales y planteamiento de una alternativa: la evaluación formativa y compartida. Retos: nuevas tendencias en educación física, deporte y recreación, (10), 31-41. Recuperado de: http://www. retos.org/numero_9_10/retos10-3.pdf.

Martínez Miguélez, M. (2007). Conceptualización de la transdisciplinariedad. Polis. Revista Latinoamericana, (16), 1-20. Recuperado de: https://journals.openedition.org/polis/4623.

Mendoza Molina, X., \& Bernabeu Tamayo, M. D. (2006). Aprendizaje basado en problemas competencias del profesional de la salud. Innovación educativa, 6(35), 1-12. Recuperado de: http:// www.redalyc.org/pdf/1794/179420847008.pdf.

Navas, J. L. (2004). La educación como objeto de conocimiento. El concepto de educación. En Pozo Andrés, M., Álvarez Castillo, J.L., Luengo Navas, J., \& Otero Urtza, E. (Eds.), Teorias e instituciones contemporáneas de educación (pp. 45-60). Madrid: Biblioteca Nueva. Recuperado de: http:// psicologiavirtual.com.mx/Aula1/Biblioteca/ Educacion/1-EducacionConcepto.pdf.

Ortiz Hernández, E. (2007). La autoevaluación estudiantil. Una práctica olvidada. Cuaderno de Investigación en la Educación. Centro de Investigaciones Educativas, (22), 107-119. Recuperado de: https://revistas. upr.edu/index.php/educacion/article/view/13269.
Pastor, V. M. L., Brunicardi, D. P., Arribas, J. C. M., \& Aguado, R. M. (2016). Los retos de la Educación Física en el Siglo XxI. Retos: nuevas tendencias en educación física, deporte y recreación, (29), 182187. Recuperado de: https://dialnet.unirioja.es/ servlet/articulo?codigo $=5400869$.

Pérez, C. R. (2003). Paradigma de la complejidad, modelos científicos y conocimiento educativo. Ágora digital, 6, 1-10. Recuperado de: http:// rabida.uhu.es/dspace/handle/10272/3518.

Ravela, P. (2016). Fichas didácticas para comprender las evaluaciones educativas. Recuperado de: http:// dide.minedu.gob.pe/handle/123456789/4999.

Rodríguez, M. (2017). Currículum, educación y cultura en la formación docente del siglo xxi desde la complejidad. Revista Educación y Humanismo, 19(33), 425-440. doi: http://dx.doi.org/10.17081/ eduhum.19.33.2654.

Ruano, J. C., Morillo, M. M., \& González, F. J. Á. (2018). Educación transdisciplinar: formando en competencias para el buen vivir. Ensaio: Avaliação e Políticas Públicas em Educação, 26(100), 619-644. doi: https://doi.org/10.1590/ S0104-40362018002601487.

Soto, C. Á., \& Vargas, J. J. L. (2019). Cuerpo, corporeidad y educación: una mirada reflexiva desde la Educación Física. Retos: nuevas tendencias en educación física, deporte y recreación, (35), 413-421. Recuperado de: https://dialnet.unirioja.es/servlet/ articulo? codigo $=6761710$. 